\title{
CALCULATION OF THE VIBRONIC TRANSITION MOMENT WHEN THE GEOMETRIES OF THE COMBINING STATES OF THE MOLECULES ARE SIGNIFICANTLY DIFFERENT
}

\author{
L. A. Gribov
}

UDC 544.174.2/.3

The concept of the adiabatic approximation is introduced from a few new standpoints, and the conditions are refined under which we can assume that the total energy of an electronic-vibrational (vibronic) state is the sum of the energies of the "electronic" and the "nuclear" problems and the wave function is represented as the product of the corresponding functions. An expression exactly corresponding to such an approximation is considered for the optical transition matrix element, and its individual terms are analyzed for any change in the geometric structure of the molecule upon optical excitation.

Key words: adiabatic approximation, optical transition probability.

Formulation of the problem. When calculating the probabilities of optical transitions between electronic- vibrational energy levels in polyatomic molecules, in the theory of spectra we typically use the Franck-Condon approximation, in which the electronic wave functions are assumed to be generally independent of the motion of the nuclei, and (significantly more rarely) the Herzberg-Teller approximation, where such motions are partially taken into account.

There is still some methodological interest in examining this problem, obtaining and analyzing all the component expressions for the transition matrix element exactly satisfying the adiabatic principle for solution of electronic-vibrational problems and in addition taking into account the possibility of a significant change in the geometry of the molecule during optical excitation.

The adiabatic approximation. All the computational algorithms employed in widely used software packages are based on the adiabatic approximation. Usually it is assumed that this approximation involves separate solution of two problems: the problem of the states of the electrons in the field of fixed Coulomb centers (the nuclei), and the problem of the states of the nuclei in some space with a specified potential function. The problem of substantiating such an approach is quite fundamental. Unfortunately, there is considerable confusion connected with this point in the literature. So it is useful to focus on it in more detail.

The first problem (we will call it the electronic problem) is solved in a system of conventional cartesian coordinates based on fundamental (Coulomb) interactions. In solving the second problem, we use various coordinate systems (cartesian, internal, etc.). In the potential function of the second problem, the fundamental interactions are not present in explicit form and are replaced by parameters of a different kind: Hooke's law elasticities (in the harmonic approximation), etc. If the relative positions of the nuclei change, then the eigennumbers $E_{\mathrm{E}}$ and the eigenfunctions $\psi_{\mathrm{E}}$ for the electronic problem depend parametrically on the nuclear coordinates. In this case, the energy of Coulomb repulsion of the nuclei at their selected relative positions is added (as an additive term) to the values of the eigennumbers (energy levels) of the purely electronic problem of electrons in the field of the fixed nuclei. Obviously such a problem can be mathematically formulated and solved without any restrictions. Note that there is no need to insist on the condition that the masses of the electrons are small compared with the masses of the nuclei. The problem of nuclear motions (or their stationary states) can be independently formulated and solved if we introduce some potential function that is dependent on the nuclear coordinates. We will not dwell on how both these problems can be mathematically solved in the general case, but rather only point out that in principle it is always possible. The problem involves working with the solution obtained, while pursuing the ultimate goal: comparison with experiment.

V. I. Vernadskii Institute of Geochemistry and Analytical Chemistry, Russian Academy of Sciences, 19 ul. Kosygina, GSP-1, Moscow 119991, Russia. E-mail: gribov@geokhi.ru. Translated from Zhurnal Prikladnoi Spektroskopii, Vol. 73, No. 3, pp. 290-293, May-June, 2006. Original article submitted December 2, 2005. 
If from the very beginning we consider the entire molecule, where the electrons and the nuclei make up an indivisible system, then we must formulate a general equation for the combined state of the electrons and the nuclei. In this case, an independent solution of the two problems for the generalized system is possible only when the operator (the Hamiltonian $\mathrm{H}_{\mathrm{EN}}$ ) is reduced to a sum of operators, each of which depends only on "its own" coordinates. It is specifically in this case that the variables are separated. Such a procedure (for an operator of general form $\mathrm{H}_{\mathrm{EN}}$ ) cannot be carried out in the general case, since the "coupling" term corresponding to electronic- nuclear interactions is not eliminated.

Let us consider the total Hamiltonian for the electronic-nuclear problem:

$$
\hat{\mathrm{H}}_{\mathrm{EN}}=\hat{\mathrm{T}}_{\mathrm{E}}+V_{\mathrm{EE}}+V_{\mathrm{EN}}+V_{\mathrm{NN}}+\hat{\mathrm{T}}_{\mathrm{N}} \text {. }
$$

Nothing is changed if we rewrite this as:

$$
\hat{\mathrm{H}}_{\mathrm{EN}}=\hat{\mathrm{T}}_{\mathrm{E}}+V_{\mathrm{EE}}+V_{\mathrm{EN}}+V_{\mathrm{NN}}+\hat{\mathrm{T}}_{\mathrm{N}}-W+W=\left(\hat{\mathrm{H}}_{\mathrm{N}}-W\right)+\left(\hat{\mathrm{T}}_{\mathrm{N}}+W\right)=\left(\hat{\mathrm{H}}_{\mathrm{E}}-W\right)+\hat{\mathrm{H}}_{\mathrm{N}} \cdot
$$

Here $\hat{\mathrm{T}}_{\mathrm{E}}$ and $\hat{\mathrm{T}}_{\mathrm{N}}$ are the kinetic operators; $V_{\mathrm{EE}}, V_{\mathrm{EN}}, V_{\mathrm{NN}}$ are the potential functions for electron-electron and other interactions; $W$ is a potential function selected for independent formulation of the problem of nuclear motions. If the problem of the states of electrons in the field of fixed nuclei is also solved independently for different nuclear positions, then we should have

$$
\hat{\mathrm{H}}_{\mathrm{E}}(Q) \psi_{\mathrm{E}}(Q)=\left(\hat{\mathrm{T}}_{\mathrm{E}}+V_{\mathrm{EE}}+V_{\mathrm{EN}}+V_{\mathrm{NN}}\right) \psi_{\mathrm{E}}(Q)=E_{\mathrm{E}}(Q) \psi_{\mathrm{E}}(Q)
$$

Let us restrict ourselves to small deviations from the initial point, assuming that it coincides with the minimum of the potential selected in formulation of the problem of the nuclear states. Then we can write: $E_{\mathrm{E}}(Q)=E_{\mathrm{E}}(0)+\Delta E_{\mathrm{E}}(Q)$ and $\psi_{\mathrm{E}}(Q)=\psi_{\mathrm{E}}(0)+\left(\partial \psi_{\mathrm{E}} / \partial Q\right)_{0} Q$ Here the $Q$ are the coordinates describing the positions of the nuclei relative to each other.

Let us consider the action of the operator $\hat{\mathrm{H}}_{\mathrm{EN}}$ on the product of the functions $\psi_{\mathrm{E}}(Q) \psi_{\mathrm{N}}(Q)$. Assuming that the function $\psi_{\mathrm{N}}(Q)$ corresponds to the operator $\hat{\mathrm{H}}_{\mathrm{N}}=\hat{\mathrm{T}}_{\mathrm{N}}+W$ and considering that the operator $\hat{\mathrm{T}}_{\mathrm{E}}$ calls for differentiation only with respect to the electronic coordinates, we obtain:

$$
\begin{gathered}
\hat{\mathrm{H}}_{\mathrm{EN}}\left[\psi_{\mathrm{E}}(Q) \psi_{\mathrm{N}}(Q)\right]=\left(\hat{\mathrm{H}}_{\mathrm{E}}-W+\hat{\mathrm{H}}_{\mathrm{N}}\right)\left[\psi_{\mathrm{E}}(Q) \psi_{\mathrm{N}}(Q)\right]=\left(E_{\mathrm{E}}(0)+\Delta E_{\mathrm{E}}(Q)\right)\left[\psi_{\mathrm{E}}(Q) \psi_{\mathrm{N}}(Q)\right]-W\left[\psi_{\mathrm{E}}(Q) \psi_{\mathrm{N}}(Q)\right]+ \\
+\hat{\mathrm{H}}_{\mathrm{N}}\left[\psi_{\mathrm{E}}(Q) \psi_{\mathrm{N}}(Q)\right] .
\end{gathered}
$$

We see that the expression is simplified if we assume $W=\Delta E_{\mathrm{E}}(Q)$. Then

$$
\hat{\mathrm{H}}_{\mathrm{EN}}\left[\psi_{\mathrm{E}}(Q) \psi_{\mathrm{N}}(Q)\right]=\left(E_{\mathrm{E}}(0)+E_{\mathrm{N}}\right)\left[\psi_{\mathrm{E}}(Q) \psi_{\mathrm{N}}(Q)\right]+2 \psi_{\mathrm{E}}^{\prime}(Q) \psi_{\mathrm{N}}^{\prime}(Q) .
$$

(The primes denote the first derivatives with respect to the relative nuclear coordinates.) The parameter $E_{\mathrm{N}}$ refers back to the problem of the motion of nuclei with Hamiltonian $\hat{\mathrm{H}}_{\mathrm{N}}=\hat{\mathrm{T}}_{\mathrm{N}}+\Delta E_{\mathrm{E}}(Q)$. We consider that action of the operator $\mathrm{T}_{\mathrm{N}}$ on a function means double differentiation with respect to the coordinates $Q$ relative to the positions of the nuclei. Therefore we can conclude that the function $\psi_{\mathrm{E}}(Q) \psi_{\mathrm{N}}(Q)$ satisfies the Schrodinger equation with Hamiltonian $\hat{\mathrm{H}}_{\mathrm{EN}}$, but only up to an additional term containing the first derivatives of the functions $\psi_{\mathrm{E}}(Q)$ and $\psi_{\mathrm{N}}(Q)$ with respect to the coordinates $Q$.

However, we found that this term does not make a contribution to the average energy:

$$
\left.\overline{\mathrm{H}}=\int \psi_{\mathrm{E}} \psi_{\mathrm{N}}\left(\hat{\mathrm{H}}_{\mathrm{EN}} \psi_{\mathrm{E}} \psi_{\mathrm{N}}\right) d v_{\mathrm{E}} d v_{\mathrm{N}}=\left[E_{\mathrm{E}}(0)+E_{\mathrm{N}}\right]+2 \int \psi_{\mathrm{E}}(Q) \psi_{\mathrm{E}}^{\prime}(Q) d v_{\mathrm{E}}\right) \psi_{\mathrm{N}} \psi_{\mathrm{N}}^{\prime} d v_{\mathrm{N}}
$$

We assume, as is conventional in quantum chemistry, that the functions $\psi_{\mathrm{E}}$ and $\psi_{\mathrm{N}}$ are real. For the functions $\psi_{\mathrm{E}}(Q)$, independently of $Q$, we have the normalization $\int \psi_{\mathrm{E}}^{2}(Q) d v_{\mathrm{E}}=1$. Therefore the derivative of this integral with respect 
to $Q$, having the form $2 \int \psi_{\mathrm{E}}(Q) \psi_{\mathrm{E}}^{\prime}(Q) d v_{\mathrm{E}}$, is equal to zero. Thus although the sum $E_{\mathrm{EN}}=E_{\mathrm{E}}(Q)+E_{\mathrm{N}}$ and the function $\psi_{\mathrm{E}}(Q) \psi_{\mathrm{N}}(Q)$ do not correspond to the Schrodinger equation with Hamiltonian $\hat{\mathrm{H}}_{\mathrm{EN}}$, the value of the average energy for the stipulations made above can be assumed to be equal to $E_{\mathrm{EN}}=E_{\mathrm{E}}(0)+E_{\mathrm{N}}$, i.e., fully corresponding to the determined separation solution of the two problems: the electronic problem and the nuclear problem. In fact, this conclusion is also the basis for all quantum chemical calculations.

Let us also point out that if $\psi_{\mathrm{E}}(Q)$ depends only linearly on $Q$, then the function $\Delta E_{\mathrm{E}}(Q)$ should be quadratic, i.e., should correspond to a harmonic potential.

The reasoning presented above shows that the adiabatic approximation is not a rigorous solution of the electronic-nuclear problem, requiring separation of variables, but rather is some model representation that is rather close to the desired solution.

Let us turn our attention to the fact that if all the functions $\psi_{\mathrm{E}}$ are eigenfunctions of the equation with the operator $\mathrm{H}_{\mathrm{E}}$ and are orthogonal for any relative positions of the nuclei (values of the coordinates $Q$ ), then the product of the functions no longer corresponds to the same equation, since for each electronic state, the potential $W$ is selected independently of the other. Nevertheless, this does not destroy the orthogonality of the functions in the form of the products $\psi_{\mathrm{E}} \psi_{\mathrm{N}}$, since it is ensured by the orthogonality of the cofactors $\psi_{\mathrm{E}}$.

It is interesting to note that if we go from the operator formulation of quantum mechanics to the matrix formulation, then in the adiabatic approximation under consideration the energy matrix becomes strictly diagonal, while the unit matrix equation describes all the stationary states.

Note the use of the same rule for calculating $E_{\mathrm{EN}}$ and $\psi_{\mathrm{EN}}$ even when the adiabatic separation of the problems based on the Schrodinger equation is already incorrect (for example, in analysis of anharmonic vibrations of polyatomic molecules). Clearly in this case we do not obtain an exact expression expression for $E_{\mathrm{EN}}$ but rather one that is an even poorer approximation (in the mathematical sense). Generally this is not pointed out.

Matrix element for an optical electronic-vibrational transition. The electronic-vibrational functions $\psi_{\mathrm{EN}}=$ $\psi_{\mathrm{E}} \psi_{\mathrm{N}}$ obtained by the rule described above are orthonormalized, although the cofactors $\psi_{\mathrm{N}}$ are orthogonal only for "their own" electronic states. From this it follows that the probability of an electronic-vibrational transition is determined by the dipole matrix element $\langle\boldsymbol{\mu}\rangle^{\mathrm{I}, \mathrm{II}}$ (the primes denote combining states).

For clarity in the following discussion, let us restrict ourselves to the one-dimensional problem. Let there be a transition from a "well" in the ground state to a "well" in the electronically excited state. Let the minimum of the "well" in the ground state be at point 0 and let the "well" in the excited state be at point 2 . Let us denote the shift of the "well" minima as $\Delta Q$. Let us assume that $\Delta Q \neq 0$ always, since in transition from one electronic state to another, the electron density must change and the new positions of the nuclei should "readjust" with that change. Let us denote the electronic cofactors for the electronic-vibrational functions for the combining states using the symbols $\Psi^{\prime}$ and $\Psi^{\prime \prime}$, and let us denote the nuclear (vibrational) cofactors as $\chi^{\prime}$ and $\chi^{\prime \prime}$. Recall that the functions $\Psi^{\prime}$ and $\Psi^{\prime \prime}$ also depend on the nuclear coordinates, but parametrically.

The matrix element for an optical dipole transition between electronic-vibrational energy levels of the molecule has the form:

$$
\langle\boldsymbol{\mu}\rangle^{\prime \prime \prime}=\int\left(\int \Psi^{\prime} \Psi^{\prime \prime} \boldsymbol{\mu}_{\mathrm{E}} d r\right) \chi^{\prime} \chi^{\prime \prime} d Q+\int\left(\int \Psi^{\prime} \Psi^{\prime \prime} d r\right) \boldsymbol{\mu}_{\mathrm{N}} \chi^{\prime} \chi^{\prime \prime} d Q
$$

The integration is carried out over the electronic $(d r)$ and nuclear $(d Q)$ coordinates. The functions $\Psi^{\prime}$ and $\Psi^{\prime \prime}$, corresponding to the same operator $\hat{\mathrm{H}}_{\mathrm{E}}$ of the electronic problem, are structurally orthogonal. Therefore the second term in the expression for $\langle\boldsymbol{\mu}\rangle^{\prime \prime \prime}$ vanishes.

Let us write the parameter $\langle\boldsymbol{\mu}\rangle_{\mathrm{E}}^{\prime \prime \prime}=\int \Psi^{\prime} \Psi^{\prime \prime} \boldsymbol{\mu}_{\mathrm{E}} d r$ in the form of a series about the point 0 with respect to the vibrational coordinates, making using of difference representations of the first and second derivatives with respect to the vibrational coordinates and limiting ourselves to only the quadratic terms:

$$
\int\left(\int \Psi^{\prime} \Psi^{\prime \prime} \boldsymbol{\mu}_{\mathrm{E}} d r\right)=\int\left(\Psi_{0}^{\prime} \Psi_{0}^{\prime \prime} \boldsymbol{\mu}_{\mathrm{E}}\right) d r+2 Q(\Delta Q)^{-1} \int \boldsymbol{\mu}_{\mathrm{E}}\left(\Psi_{1}^{\prime} \Psi_{1}^{\prime \prime}-\Psi_{0}^{\prime} \Psi_{0}^{\prime \prime}\right) d r+2 Q^{2}(\Delta Q)^{-2} \int \boldsymbol{\mu}_{\mathrm{E}}\left(\Psi_{2}^{\prime} \Psi_{2}^{\prime \prime}-2 \Psi_{1}^{\prime} \Psi_{1}^{\prime \prime}+\Psi_{0}^{\prime} \Psi_{0}^{\prime \prime}\right) d r
$$




$$
=\langle\boldsymbol{\mu}\rangle_{0}^{\prime \prime \prime}+2 Q(\Delta Q)^{-1}\left(\langle\boldsymbol{\mu}\rangle_{1}^{\prime \prime \prime}-\langle\boldsymbol{\mu}\rangle_{0}^{\prime \prime \prime}\right)+2 Q^{2}(\Delta Q)^{-2}\left(\langle\boldsymbol{\mu}\rangle_{2}^{\prime \prime \prime \prime}-2\langle\boldsymbol{\mu}\rangle_{1}^{\prime \prime \prime}+\langle\boldsymbol{\mu}\rangle_{0}^{\prime \prime \prime}\right) .
$$

We see that the first term corresponds to a Franck-Condon transition from a state of the minimum in the lower potential "well". In the second term, there is a term corresponding to a vertical electronic transition from an intermediate point 1 in the lower state to the same point in the upper state. In the third term, there is an analogous component, but now for point 2 (the minimum of the "well" for the excited state).

Note one very important point that usually is not discussed. All the calculations are done selecting the minimum of the "well" in the lower state as the initial point, i.e., for absorption. In the case of emission, it is reasonable to proceed similarly, i.e., to select as the initial point the minimum of the "well" for the excited state. In this case, the matrix element $\langle\boldsymbol{\mu}\rangle_{\mathrm{E}}^{\prime \prime \prime}$ (transition downward) turns out to not be equal to the matrix element $\langle\boldsymbol{\mu}\rangle_{\mathrm{E}}^{\prime \prime \prime}$ (transition upward)! This contradicts the fundamental assumption of the theory of interaction between light and a quantum system, that the matrix element for transition between any energy levels is independent of the initial state. Note that the integral of general form $\langle\boldsymbol{\mu}\rangle_{\mathrm{E}}^{\prime \prime \prime}=\int \Psi^{\prime} \Psi^{\prime \prime} \boldsymbol{\mu}_{\mathrm{E}} d r$ does not depend on the sequential order of $\Psi^{\prime}$ and $\Psi^{\prime \prime}$. The dependence is apparent as we go to approximations connected with expansions in a series about different points.

We can satisfy the requirement that the matrix elements for absorption and emission be equal if as $\langle\boldsymbol{\mu}\rangle_{\mathrm{E}}^{\prime \prime \prime}$ we take the average value, i.e., if we assume $\left(\langle\boldsymbol{\mu}\rangle_{\mathrm{E}}^{\prime \prime \prime}\right)_{\mathrm{avg}}=\frac{\langle\boldsymbol{\mu}\rangle_{\mathrm{E}}^{\prime \prime \prime}+\langle\boldsymbol{\mu}\rangle_{\mathrm{E}}^{\prime \prime \prime}}{2}$. For small $\Delta Q$, this may prove to be unimportant, but for significant values (such situations are quite likely) we need to go to the proposed model expression.

The final expression for the matrix element $\langle\boldsymbol{\mu}\rangle^{\prime \prime \prime}$ is obtained after integration over the nuclear coordinates. Then the parameter $\langle\boldsymbol{\mu}\rangle_{0}^{\prime \prime \prime}$ is replaced by $\langle\boldsymbol{\mu}\rangle_{0}^{\prime \prime \prime} \int \chi^{\prime} \chi^{\prime \prime} d Q$, and in the next two terms instead of $Q$ and $Q^{2}$ we have respectively the matrix elements $\int \chi^{\prime} Q \chi^{\prime \prime} d Q$ and $\int \chi^{\prime} Q^{2} \chi^{\prime \prime} d Q$. For small shifts $\Delta Q$ and small changes in the curvature of the "well" for the excited state compared with the "well" for the ground state, we can set $\int \chi^{\prime} \chi$ "d $Q=1$, while the integrals $\int \chi^{\prime} Q \chi^{\prime \prime} d Q$ and $\int \chi^{\prime} Q^{2} \chi^{\prime \prime} d Q$ are assumed to be equal to the matrix element for the normal coordinate and its square.

Obviously such an approximation is no longer suitable if $\Delta Q$ is rather large and if the parameters of the "wells" are considerably different. For the one-dimensional case, we can make use of analytical formulas for such integrals, but in the general multidimensional case we need to go to numerical methods.

Polyatomic molecules. Let us consider the general case of a polyatomic molecule. Then we need to go to the multidimensional space of the normal coordinates. As far as we know, the resulting problem was discussed for the first time only in [1-3].

Recall that in solving the electronic-vibrational problem, each vibrational problem is formulated and solved independently of the other. In particular, for the ground and excited electronic states, to which different "wells" are assigned, we obtain a different set of normal coordinates. Let us denote them as $\mathbf{Q}^{\prime}$ and $\mathbf{Q}^{\prime \prime}$ (the primes mark the different sets of normal coordinates). It is important to consider that both the geometric properties of the electronic term (the position of the minimum) and the curvature parameters of the "well" are reflected in the normal coordinates.

We have shown that there is a relationship $\mathbf{Q}^{\prime \prime}=\mathbf{A} \mathbf{Q}^{\prime}+\mathbf{b}$ between the sets of coordinates $\mathbf{Q}^{\prime}$ and $\mathbf{Q}^{\prime \prime}$. Here the matrix $\mathbf{A}$ defines the rotation and the change in the scales of the coordinates $\mathbf{Q}^{\prime \prime}$ relative to the coordinates $\mathbf{Q}^{\prime}$, while the b column defines the shift of the origin for the normal coordinates $\mathbf{Q}^{\prime \prime}$ relative to $\mathbf{Q}^{\prime}$ ( $\mathbf{b}$ is a vector in the multidimensional space, drawn from the minimum of the initial potential "well" to the minimum of the "well" in the combining state). It is important that both the matrix $\mathbf{A}$ and the vector $\mathbf{b}$ can be calculated for molecules of any complexity for any change in the geometric structures of the excited states and characteristics of the "wells".

If we take all this into account, then we can conclude that the integral $\int \chi^{\prime} \chi^{\prime \prime} d \mathbf{Q}$ becomes multidimensional and rather complex from the calculation standpoint [4]. 
In the second term in the formula for $\int \Psi^{\prime} \Psi^{\prime \prime} \boldsymbol{\mu}_{\mathrm{E}} d r$, we write the sum $\left.\sum_{k} \frac{2\left(\langle\boldsymbol{\mu}\rangle_{1 k}^{\prime \prime \prime}-\langle\boldsymbol{\mu}\rangle_{0}^{\prime \prime \prime}\right.}{b_{k}}\right)_{Q_{k}^{\prime}}$. Here $b_{k}$ is the column b matrix element. Let us calculate the matrix elements $\langle\boldsymbol{\mu}\rangle_{1 k}^{\prime \prime \prime}$ for points corresponding to shifts of the minimum for the "wells" along the $Q_{k}$ directions by the distance $b_{k} / 2$. The index $\mathrm{k}$ refers to the normal coordinates.

The third term for the matrix element of an optical electronic-vibrational transition in the multidimensional case takes on the form:

$$
2 \sum_{k} Q_{k}^{\prime 2}\left(b_{k}^{2}\right)^{-1}\left(\langle\boldsymbol{\mu}\rangle_{2 k}^{\prime \prime \prime}-2\langle\boldsymbol{\mu}\rangle_{1 k}^{\prime \prime \prime}+\langle\boldsymbol{\mu}\rangle_{0}^{\prime \prime \prime}\right)+2 \sum_{\substack{k, n \\ k \neq n}} Q_{k}^{\prime} Q_{n}^{\prime}\left(b_{k} b_{n}\right)^{-1}\left(\langle\boldsymbol{\mu}\rangle_{1 k, 1 n}^{\prime \prime \prime}-\langle\boldsymbol{\mu}\rangle_{1 k}^{\prime \prime \prime}-\langle\boldsymbol{\mu}\rangle_{1 n}^{\prime \prime \prime \prime}+\langle\boldsymbol{\mu}\rangle_{0}^{\prime \prime \prime}\right)
$$

The symbols $1 k, 2 k$ mean that the matrix elements are calculated at the points where all the coordinates $Q_{n(n \neq k)}$ correspond to the initial point 0 , while the coordinate $Q_{k}$ runs for point 1 etc.

In subsequent integration over the vibrational coordinates, the integrals $\int \chi^{\prime}\left(\mathbf{Q}^{\prime} Q_{k}^{\prime} \chi^{\prime \prime}\left(\mathbf{Q}^{\prime \prime}\right) d \mathbf{Q}^{\prime}\right.$ and $\left.\int \chi^{\prime}\left(\mathbf{Q}^{\prime}\right) Q_{k}^{\prime} Q_{n}^{\prime} \chi^{\prime \prime} \mathbf{Q}^{\prime \prime}\right) d \mathbf{Q}^{\prime \prime}$ appear. They can also be calculated using the approach proposed in [4].

Thus the general calculation algorithm is formulated and is completely solved both in this sense of the problem and for the multidimensional case. Another point is that the procedure becomes rather unwieldy and leads to considerable complication of the computer software. A simplification can be achieved by selecting an approximation that is suitable for practical application, which may be done just on the basis of the corresponding computer experiments.

This work was done with the financial support of a grant from the President of the Russian Federation (No. NSh-1186.2003.3) and a grant from the Russian Foundation for Basic Research (project No. 04-03-32087a).

\section{REFERENCES}

1. L. A. Gribov, Opt. i Spektr., 59, No. 6, 1337-1341 (1985).

2. L. A. Gribov, Dokl. Akad. Nauk SSSR, 292, No. 5, 1161-1165 (1987).

3. L. A. Gribov and N. I. Prokof'eva, Zh. Prikl. Spektr., 46, No. 4, 603-608 (1987).

4. L. A. Gribov, Dokl. Ross. Akad. Nauk, 404, No. 1, 54-56 (2005). 